\title{
A DYNAMIC AND STOCHASTIC EXTENSION OF THE MAIN THEOREMS OF INTERNATIONAL TRADE: THE CASE OF EXHAUSTIBLE AND NON- RENEWABLE FACTORS
}

Francisco Venegas-Martínez*

Tecnologico de Monterrey, Campus Ciudad de México

(Received 2 August 2001, accepted 3 January 2003)

\begin{abstract}
Most of the models of international trade are static and deterministic, factors of production offer services in a given an steady flow for ever and ever. In this paper we provide some extensions to the main theorems in international trade (Ribczynski, Stolper-Samuelson, Hecksher-Ohlin-Samuelson and Factor Price Equalization). We consider exhaustible and nonrenewable resources in a dynamic framework where factors and extraction rates are driven by stochastic differential equations following a given trend. In this case, stochastic disturbances such as: market shocks or unexpected increases or decreases in reserves, are modeled through the Brownian motion (the continuous version of a random walk).
\end{abstract}

\section{Resumen}

La mayoría de los modelos de comercio internacional son estáticos y deterministas, los factores de la producción se presentan a una tasa dada que permanece constante para siempre. En este trabajo proporcionamos algunas extensiones a los teoremas fundamentales del comercio internacional (Ribczynski, Stolper-Samuelson, Hecksher-Ohlin-Samuelson y el de igualación de precios de los factores). Consideramos recursos que se agotan y no son renovables en un marco dinámico donde los factores y las tasas de extracción se comportan de acuerdo a ecuaciones diferenciales estocásticas que siguen una tendencia dada. En este caso, pertubaciones estocásticas como: choques de mercado o aumentos o disminuciones inesperadas de reservas, son modelados a través del movimiento Browniano (la versión continua de una caminata aleatoria).

JEL Classification: F11

Keywords: International Trade

* Centro de Investigación en Finanzas, Tecnológico de Monterrey, Campus Ciudad de México, Calle del Puente 222, Aulas 3, Cuarto piso, Col. Ejidos de Huipulco, Del. Tlalpan, 14380 México, D. F., Telephone +52(55)54831861. E-mail: fvenegas@itesm.mx

The author is grateful to the anonymous referees for several comments and observations. A number of useful suggestions were provided by Thomas Lowinger. 


\section{Introduction}

The main theorems of international trade (Ribczynski, Stolper-Samuelson, Hecksher-Ohlin-Samuelson, and Factor Price Equalization) provide long-run and steady-state results. The factors of production (Ricardian Factors) offer a service in a given an steady flow for ever and ever. A very natural question arise: Is it possible to develop dynamic and stochastic counterparts to include exhaustible and non-renewable or renewable factors (Hotelling factors)? such as mineral and oil reserves. This is certainly the case of México and other oil producing developing countries. We shall develop a number of models to deal with this situation.

A few models of international trade that consider exhaustible resources are available in the literature, we mention, for instance, Kemp and Long (1979) and (1982), and Tawada (1982). However, it is missing a more realistic dynamic framework to analyze international trade in the presence of non-renewable resources subject to unexpected stochastic disturbances. In this research, we provide a dynamic and stochastic extensions to the main theorems in international trade in a richer framework where stochastic disturbances such as: market shocks, unexpected increases or decreases in reserves, earthquakes or floods, etc, are modeled through the Brownian motion.

The structure and development of this paper is as follows: In section 2, we shall present a first model for two exhaustible and non-renewable factors. The assumptions made are as close as possible to the Hecksher-Ohlin-Samuelson theorem. Through section 3, we shall state the conditions for market efficiency for dynamic models. In section 4, we incorporate into the model the demand side and state the full model. Through section 5 we develop a model with one exhaustible and non-renewable factor and one Ricadian factor; labor. In section 6 , we study the case for renewable resources. Through section 7 , factors and extraction rates follow a given trend, but stochastic disturbances, driven by the Brownian motion, may occur. Finally, in section 8 , we give a number of comments on the advantages and delimitations of our work.

\section{A First Model}

The assumptions that we made in this section, for a small and open economy, are as close as possible to those in the Hecksher-Ohlin-Samuelson theorem:

(i) There are two goods, $x(t)$ and $y(t)$;

(ii) There are two exhaustible and non-renewable factors, $R(t)$ and $S(t)$ (Hotelling factors). These paths are predetermined;

(iii) The initial stocks $R(0)$ and $S(0)$ are given and positive;

(iv) The rates of extraction of $R(t)$ and $S(t)$ are $r(t)$ and $s(t)$, respectively, satisfy:

$$
\left\{\begin{array}{l}
\dot{R}(t)=-r(t), \\
\dot{S}(t)=-s(t) .
\end{array}\right.
$$

The transversality conditions are given by:

$$
\lim _{t \rightarrow \infty} R(t)=0 \quad \text { and } \quad \lim _{t \rightarrow \infty} S(t)=0 .
$$


We shall come back later to these assumptions to deal with a more realistic situation in which extraction rates follow an exponential pattern, but stochastic disturbances may occur;

(v) The production functions for both industries are homogeneous of degree one, strictly quasi-concave and such that both factors are indispensable. These technologies are represented by:

$$
\left\{\begin{array}{l}
x(t)=F\left(r_{x}(t), s_{x}(t)\right) \\
y(t)=G\left(r_{y}(t), s_{y}(t)\right)
\end{array}\right.
$$

(vi) The two industries differ in their relative "factor intensities". Suppose for instance that

$$
k_{y}(t)=\frac{r_{y}(t)}{s_{y}(t)}>\frac{r_{x}(t)}{s_{x}(t)}=k_{x}(t)
$$

(vii) There are no production costs;

(viii) There are no distortions;

(ix) There is perfect competition in all markets;

(x) The economy is small and open (price-taking behavior).

\section{Market Efficiency}

In this section, in a dynamic approach, we deal with de market efficiency conditions. Given $r(t)$ and $s(t)$ the way in which $r_{x}(t), r_{y}(t), s_{x}(t)$ and $s_{y}(t)$ are combined in the production processes is obtained by solving the following optimal control problem:

$$
\text { Maximize } \int_{0}^{\infty} e^{-i t} F\left(r_{x}(t), s_{x}(t)\right) \mathrm{d} t
$$

$$
\text { subject to : } \int_{0}^{\infty} e^{-i t} G\left(r(t)-r_{x}(t), s(t)-s_{x}(t)\right) \mathrm{d} t=\text { constant, }
$$

where $i>0$ is the interest rate. The Lagrangian associated, with the above optimization problem, is given by

$$
L\left(r_{x}(t), s_{x}(t), \lambda\right)=\left[F \left(r_{x}(t), s_{x}(t)+\lambda G\left(r(t)-r_{x}(t), s(t)-s_{x}(t)\right] e^{-i t}\right.\right.
$$

and the first order conditions are, i.e., necessary conditions, are given by (see Appendix):

$$
\left\{\begin{array}{l}
F_{r_{x}}-\lambda G_{r_{y}}=0 \\
F_{s_{x}}-\lambda G_{s_{y}}=0
\end{array}\right.
$$

this in turns, implies that

$$
\frac{F_{r_{x}}}{F_{s_{x}}}=\frac{G_{r_{y}}}{G_{s_{y}}}
$$


equivalently, in terms of the marginal rates of technical substitution,

$$
M R T S_{r, s}^{x}=M R T S_{r, s}^{y}
$$

Notice also that

$$
\left\{\begin{array}{l}
\mathrm{d} y(t)=G_{r_{y}} \mathrm{~d} r_{y}+G_{s_{y}} \mathrm{~d} s_{y} \\
\mathrm{~d} x(t)=-F_{r_{x}} d r_{y}-F_{s_{x}} \mathrm{~d} s_{y}
\end{array}\right.
$$

from where

$$
\begin{aligned}
\mathrm{d} y(t) & =G_{r_{y}}\left(-\frac{1}{F_{r_{x}}} \mathrm{~d} x(t)-\frac{F_{s x}}{F_{s_{x}}} \mathrm{~d} s_{y}\right)+G_{s_{y}} \mathrm{~d} s_{y} \\
& =-\frac{G_{r_{y}}}{F_{r_{x}}} \mathrm{~d} x(t)-G_{r_{y}}\left(M R T S_{r, s}^{y}-M R T S_{r, s}^{x}\right) \mathrm{d} s_{y} \\
& =-\frac{G_{r_{y}}}{F_{r_{x}}} \mathrm{~d} x(t) .
\end{aligned}
$$

Thus,

$$
M R T_{x, y}=-\frac{\mathrm{d} y(t)}{\mathrm{d} x(t)}=-\frac{G_{r_{y}}}{F_{r_{x}}}<0,
$$

which is the slope of the production possibility curve.

On the other hand, given

$$
\left\{\begin{array}{l}
r_{x}(t)=r_{x}(r(t), s(t)) \\
s_{x}(t)=s_{x}(r(t), s(t)) \\
r_{y}(t)=r_{y}(r(t), s(t)) \\
s_{y}(t)=s_{y}(r(t), s(t))
\end{array}\right.
$$

and given the terms of trade $p(t)=p_{y}(t) / p_{x}(t)$, it can be shown that there exist $\underline{e}$ and $\bar{e}$ so the maximum total output, in terms of good $x$ satisfies

$$
Y(t)=Y(r(t), s(t) \mid p(t))=\left\{\begin{array}{l}
r(t) x(1, e(t)), \quad e(t) \leq \underline{e} \\
r(t)[x(1, e(t))+p(t) y(1, e(t))], \quad \underline{e} \leq e(t) \leq \bar{e} \\
r(t) p(t) y(1, e(t)), \quad e(t) \geq \bar{e}
\end{array}\right.
$$

where $e(t)=r(t) / s(t)$. We have also made use of the fact that the production of each commodity is subject to constant returns to scale. If $e(t) \leq \underline{e}$, it is optimal to produce only $x$; if $e(t) \geq \bar{e}$, it is optimal to produce only $y$; and finally if $\underline{e} \leq e(t) \leq \bar{e}$, it is optimal to produce both goods.

\section{The First Model in Full}

Turning to the demand side we make now the following assumptions:

(i) There is a representative individual (or family). Here, the community is composed of identical individuals who wish to maximize utility for consumption, for their children, for their grand children, and for all their 
descendants. Equivalently, we can also think of an individual with infinite life;

(ii) The individual discount rate of time preference, $\rho$, is constant through time;

(iii) The individual has access to a perfectly competitive international capital market.

The maximization utility problem in full detail for a small country

$$
\begin{gathered}
\text { Maximize } \int_{0}^{\infty} e^{-\rho t} U\left(c_{x}(t), c_{y}(t)\right) \mathrm{d} t, \\
\text { subject to : }\left\{\begin{array}{l}
\int_{0}^{\infty} e^{-i t}\left[c_{x}(t)+p(t) c_{y}(t)\right] \mathrm{d} t=\int_{0}^{\infty} e^{-i t} Y(s(t), r(t) \mid p(t)) \mathrm{d} t \\
\dot{R}(t)=-r(t), \\
\dot{S}(t)=-s(t), \\
R(t), \quad S(t) \geq 0 \text { (predetermined), } \\
R(0), \quad S(0)>0 \text { (given initial stocks), } \\
r(t), \quad s(t) \geq 0 .
\end{array}\right.
\end{gathered}
$$

The first constraint says that the present value of consumption equals the present value of the stream of produced output. It is important to point out that

$$
Y(0)-\lim _{t \rightarrow \infty} Y(t) e^{-i t}=\int_{0}^{\infty}[i Y(t)-\dot{Y}(t)] e^{-i t} \mathrm{~d} t,
$$

so the constraint

$$
\int_{0}^{\infty} e^{-i t} C(t) \mathrm{d} t=\int_{0}^{\infty} e^{-i t} Y(s(t), r(t) \mid p(t)) \mathrm{d} t,
$$

where $C(t)=c_{x}(t)+p(t) c_{y}(t)$, may be rewritten as

$$
\dot{Y}(t)+Y(0)=i C(t),
$$

joint with the transversality constraint

$$
\lim _{t \rightarrow \infty} Y(t) e^{-r t}=0
$$

This is a non-Ponzy game condition, that is, the individual cannot be repaying debt with borrowing. Hence, the optimization problem can be written now as

$$
\text { Maximize } \int_{0}^{\infty} e^{-\rho t} U(C(t)) \mathrm{d} t
$$




$$
\text { subject to : }\left\{\begin{array}{l}
Y(0)+\dot{Y}(t)=i C(t), \\
\lim _{t \rightarrow \infty} Y(t) e^{-i t}=0 \\
\dot{R}(t)=-r(t), \\
\dot{S}(t)=-s(t) \\
R(t), \quad S(t) \geq 0 \text { (predetermined), } \\
R(0), \quad S(0)>0 \text { (given initial stocks), } \\
r(t), \quad s(t) \geq 0 .
\end{array}\right.
$$

Also, since

$$
R(0)-\lim _{t \rightarrow \infty} R(t) e^{-i t}=\int_{0}^{\infty}[i R(t)-\dot{R}(t)] e^{-i t} \mathrm{~d} t
$$

we may write the constraint $\dot{R}(t)=-r(t)$ in the following way;

$$
\int_{0}^{\infty}[i R(t)-\dot{R}(t)] e^{-i t} \mathrm{~d} t=R(0), \quad \text { and } \quad \lim _{t \rightarrow \infty} R(t) e^{-i t}=0 .
$$

Now, to solve the utility maximization problem, we may use the so-called Fisher Separation Theorem, in that case the country to maximize first its present value of the stream of wealth, that is,

$$
\text { Maximize } \int_{0}^{\infty} e^{-i t} Y(r(t), s(t) \mid p(t)) \mathrm{d} t
$$

$$
\text { subject to }\left\{\begin{array}{l}
\dot{R}(t)=-r(t) \\
\dot{S}(t)=-s(t) \\
R(t), \quad S(t) \geq 0 \quad \text { (predetermined), } \\
R(0), \quad S(0)>0 \text { (given initial stocks), } \\
r(t), \quad s(t) \geq 0
\end{array}\right.
$$

and then solve the problem (assuming $\rho=i$ )

$$
\text { Maximize } \int_{0}^{\infty} e^{-\rho t} U\left(c_{x}(t), c_{y}(t)\right) \mathrm{d} t,
$$

$$
\text { subject to : } \int_{0}^{\infty} e^{-i t}\left[c_{x}(t)+p(t) c_{y}(t)\right] \mathrm{d} t=\int_{0}^{\infty} e^{-i t} Y(\widehat{s}(t), \widehat{r}(t) \mid p(t)) \mathrm{d} t,
$$

where $\widehat{r}(t)$ and $\widehat{s}(t)$ are the optimal extraction rates for the previous optimization problem.

When international borrowing and lending is impossible, we proceed as follows. Notice first that given $p(t), r(t)$ and $s(t)$, then $c_{x}(t)$ and $c_{y}(t)$ may 
be expressed as functions of $Y(r(t), s(t) \mid p(t))$ so we obtain an indirect utility function $V(Y(r(t), s(t) \mid p(t)))$, and the problem to be solved is:

$$
\begin{aligned}
& \text { Maximize } \int_{0}^{\infty} e^{-\rho t} V(Y(r(t), s(t) \mid p(t)) \mathrm{d} t, \\
& \text { subject to }\left\{\begin{array}{l}
\dot{R}(t)=-r(t), \\
\dot{S}(t)=-s(t), \\
R(t), \quad S(t) \geq 0 \text { (predetermined), } \\
R(0), \quad S(0)>0 \text { (given initial stocks), } \\
r(t), \quad s(t) \geq 0 .
\end{array}\right.
\end{aligned}
$$

We proceed first with the indirect utility approach. The other approach will be studied later. The Hamiltonian, for an interior solution (since each factor is indispensable), is given by

$$
H\left(r(t), s(t), \lambda_{1}, \lambda_{2}\right)=e^{-\rho t} V(Y(r(t), s(t) \mid p(t)))-\lambda_{1} r(t)-\lambda_{2} s(t) .
$$

The first order conditions (see Appendix) for this case are given by:

$$
\left\{\begin{array}{l}
e^{-\rho t} \frac{\partial V}{\partial Y} \frac{\partial Y}{\partial r}-\lambda_{1}=0 \\
e^{-\rho t} \frac{\partial V}{\partial Y} \frac{\partial Y}{\partial s}-\lambda_{2}=0
\end{array}\right.
$$

here $\lambda_{1}, \lambda_{2}$ are positive constants. The above expressions say that the present value of the marginal social utility of each factor is constant. Thus, whenever extraction takes place

$$
\frac{\frac{\partial Y}{\partial r}}{\frac{\partial Y}{\partial s}}=\text { constant }=\frac{\lambda_{1}}{\lambda_{2}} .
$$

Therefore, while production lasts, $e(t)$ must be constant and

$$
e(t)=\frac{s(t)}{r(t)}=\text { constant }=\frac{S(0)}{R(0)}
$$

Whether the rates of extraction and output go to zero in finite time depends on the behavior of marginal utility as consumption goes to zero. Summarizing the above discussion we have

Lemma 4.1 If there is a solution of the utility maximization problem. Then as long as production lasts $e(t)=s(t) / r(t)=S(0) / R(0)$.

By a straightforward argument, we also have the following result:

Lemma 4.2 Under the assumption of a small country, the extraction declines at the rate $-\rho / \eta$, where

$$
\eta=\frac{\partial^{2} V}{\partial Y^{2}} \frac{Y}{\frac{\partial V}{\partial Y}}
$$


is the elasticity of marginal utility. That is,

$$
\left\{\begin{array}{l}
\frac{\dot{r}(t)}{r(t)}=\frac{\rho}{\eta} \\
\frac{\dot{s}(t)}{s(t)}=\frac{\rho}{\eta}
\end{array}\right.
$$

Indeed, from

$$
e^{-\rho t} \frac{\partial V}{\partial Y} \frac{\partial Y}{\partial r}-\lambda_{1}=0
$$

and noting that $Y(t)=r(t) x(1, e(t) \mid p(t))+r(t) p(t) y(1, e(t) \mid p(t))$ we get

$$
\frac{\partial Y}{\partial r}=x(1, e(t) \mid p(t))+p(t) y(1, e(t) \mid p(t))=\mathrm{constant}
$$

since optimal $e(t)$ remains constant. Therefore,

$$
\frac{\partial^{2} V}{\partial Y^{2}}\left(\frac{\partial Y}{\partial r} \dot{r}+\frac{\partial Y}{\partial s} \dot{s}\right) \frac{\partial Y}{\partial r}=\rho \lambda_{1} e^{-\rho t}=\rho \frac{\partial U}{\partial Y} \frac{\partial Y}{\partial r} .
$$

Hence,

$$
\frac{\partial^{2} V}{\partial Y^{2}} \frac{\dot{r}}{r}\left[\frac{\partial Y}{\partial r} r+\frac{\partial Y}{\partial s} s \frac{\dot{s}}{s}\left(\frac{\dot{r}}{r}\right)^{-1}\right] \frac{\partial Y}{\partial r}=\frac{\partial Y}{\partial r} \rho \lambda_{1} e^{-\rho t}=\rho \frac{\partial V}{\partial Y} \frac{\partial Y}{\partial r} .
$$

Recalling now that $Y(t)=Y(r(t), s(t) \mid p(t))$ is homogeneous of degree one in $r(t)$ and $s(t)$, we get

$$
\frac{\partial Y}{\partial r} r+\frac{\partial Y}{\partial s} s=Y,
$$

and since

$$
\frac{\dot{s}}{s}\left(\frac{\dot{r}}{r}\right)^{-1}=1,
$$

we obtain

$$
\frac{\partial^{2} U}{\partial Y^{2}} \frac{Y}{\frac{\partial U}{\partial Y}}=\frac{\dot{r}}{r}=\rho,
$$

that is

$$
\eta \frac{\dot{r}}{r}=\rho \quad \text { or } \quad \frac{\dot{r}}{r}=\frac{\dot{\rho}}{\eta} .
$$

A similar result holds for $s=s(t)$.

We now prove the following theorem

Theorem 4.1 Rybczynski Theorem for two exhaustible and non-renewable resources. Suppose both commodities are produced along an optimal path. Suppose also that there is an increase in the initial stock $R(0)$ and that $x$ is relatively intensive in the use of $R$. Then the increase in $R(0)$ entails an increase in 
$r(t) / s(t)=R(0) / S(0)$ at each $t$, so the standard Rybcynski theorem applies at each $t$. Therefore, at each point of time at which production takes place, there is an increase in the relative and absolute output of commodity $x$.

Theorem 4.2 Stolper-Samuelson theorem for two exhaustible and non-renewable factors. Suppose that $x$ is relatively intensive in the use of $R$ and that both goods are produced along an optimal path and let the same be true after an increase in $p_{x}$. Notice first that before and after the price change the ratio of factors $e(t)=R(0) / S(0)$ is independent of commodity prices. Therefore, by applying the standard Stolper-Samuelson theorem, at each point of time at which production takes place, there is an increase in the marginal product of $R$ (in each industry) and a reduction of the marginal product of $S$ (in each industry).

Theorem 4.3 Hecksher-Ohlin-Samuelson for two exhaustible and non-renewable factors. Suppose that for each of two free-trading countries preferences are identical satisfying both that $V(Y)$ is an increasing function, strictly concave with continuous second derivative which satisfies (Inada conditions)

$$
\lim _{Y \rightarrow \infty} V^{\prime}(Y)=0, \quad \lim _{Y \rightarrow 0} V^{\prime}(Y)=\infty .
$$

Hence, the individual's preferences through time are represented by

$$
W(s)=\int_{s}^{\infty} V\left((Y(t)) e^{-\rho(t-s)} \mathrm{d} t\right.
$$

so preferences are separable. To be more specific, we may consider a utility function of the form

$$
V(Y(t))= \begin{cases}\frac{Y(t)^{1-\gamma}}{1-\gamma}, & \text { for } \gamma>0, \quad \gamma \neq 1, \\ \log Y(t), & \text { for } \gamma=1,\end{cases}
$$

here we have removed the discontinuity of the function at $\gamma=1$. Observe that for this consumption function the elasticity of substitution between $Y(t)$ and $Y(s)$ with $s \neq t$, is given by

$$
\sigma[V(Y(t))]=-\frac{V^{\prime}[Y(t)] / V^{\prime}[Y(s)]}{Y(s) / Y(t)} \frac{d[Y(s) / Y(t)]}{d\left\{V^{\prime}[Y(s)] / V^{\prime}[Y(t)]\right\}}=\frac{1}{\gamma} .
$$

Also notice that when $s \rightarrow t$ the elasticity of substitution satisfies

$$
\lim _{s \rightarrow t} \sigma[V(Y(t))]=-V^{\prime}[Y(t)] /\left\{V^{\prime \prime}[Y(t)] Y(t)\right\}=\sigma=\frac{1}{\gamma},
$$

in this case de relative degree of risk aversion is

$$
-V^{\prime \prime}[Y(t)] \frac{Y(t)}{V^{\prime}[Y(t)]}=\gamma
$$


Observe also that for each country (We shall denote foreign variables through the symbol ${ }^{*}$ ) there is an optimal $e(t)=\lambda=$ constant and $e^{*}(t)=\lambda^{*}=$ constant, respectively, and

$$
\left\{\begin{array} { l } 
{ \frac { \dot { r } ( t ) } { r ( t ) } = \frac { \rho } { \eta } , } \\
{ \frac { \dot { s } ( t ) } { s ( t ) } = \frac { \rho } { \eta } , }
\end{array} \quad \text { and } \quad \left\{\begin{array}{l}
\frac{\dot{r}^{*}(t)}{r^{*}(t)}=\frac{\rho}{\eta}, \\
\frac{\dot{s}^{*}(t)}{s^{*}(t)}=\frac{\rho}{\eta},
\end{array}\right.\right.
$$

where both domestics and foreigners have the same $\rho$ and the same $\eta$ as well. Then from the static lines, as long as production continues, the country which initially is relatively well endowed with the Hotelling factor will export the commodity which is relatively intensive in its use of the factor. Furthermore, since elasticity of the marginal utility is constant and equal to $\gamma$, then there is a trading equilibrium with constant terms of trade.

Theorem 4.4 Factor Price Equalization theorem for two exhaustible and nonrenewable factors. If and only if the extractions vectors $(\widehat{r}(t), \widehat{s}(t))$ and $\left(\widehat{r}^{*}(t)\right.$, $\widehat{s}^{*}(t)$ ) lie in a common cone of diversification defined by the equilibrium (constant) terms of trade, then the present value of marginal product of each resource is the same since

$$
\left\{\begin{array}{l}
\frac{\partial V^{*}}{\partial Y^{*}} \frac{\partial Y^{*}}{\partial r^{*}}=\frac{\partial V}{\partial Y} \frac{\partial Y}{\partial r}=\lambda_{1} e^{\rho t}, \\
\frac{\partial V^{*}}{\partial Y^{*}} \frac{\partial Y^{*}}{\partial s^{*}}=\frac{\partial V}{\partial Y} \frac{\partial Y}{\partial s}=\lambda_{2} e^{\rho t}
\end{array}\right.
$$

\section{A Second Model with One Ricardian Factor an One Hotelling Factor}

In this section, we introduce a Ricardian factor, namely labor, and one Hotelling factor, which is exhaustible and non-renewable. Let us establish, first, a list of assumptions:

(i) There are two goods, $x(t)$ and $y(t)$;

(ii) There are two factors. One Ricardian factor, $L$, which is internationally immobile, and one exhaustible and non-renewable factor (Hotelling factor), $R(t)$

(iii) The initial stock $R(0)$ of $R(t)$ is positive;

(iv) The rate of extraction of $R(t), r(t)$, satisfies

$$
\dot{R}(t)=-r(t) .
$$

The transversality conditions is

$$
\lim _{t \rightarrow \infty} R(t)=0
$$


(v) The production functions for both industries are homogeneous of degree one, strictly quasi-concave and such that both factors are indispensable, and they are represented by:

$$
\begin{aligned}
& \left\{\begin{array}{l}
x(t)=F\left(L_{x}, r_{x}(t)\right), \\
y(t)=G\left(L_{y}, r_{y}(t)\right),
\end{array}\right. \\
& L_{x}+L_{y}=\bar{L} ;
\end{aligned}
$$

(vi) There are no production costs;

(vii) There are no distortions;

(viii) There is perfect competition in all markets;

(ix) The economy is small (price-taking behavior);

(x) There is no international borrowing and lending;

(xi) There is a representative individual who maximizes utility for consumption;

(xii) The individual discount rate of time preference, $\rho$, is constant through time;

(xiii) Given $p(t)$, there exist $\underline{r}$ and $\bar{r}$ such that

$$
Y(t)=Y(L, r(t) \mid p(t))=\left\{\begin{array}{l}
x(\bar{L}, r(t)), \quad r(t) \leq \underline{r}, \\
x(\bar{L}, r(t))+p(t) y(\bar{L}, r(t)), \quad \underline{r} \leq r(t) \leq \bar{r} \\
p(t) y(\bar{L}, r(t)), \quad r(t) \geq \bar{r} .
\end{array}\right.
$$

If $r(t) \leq \underline{r}$ it is optimal to produce only $x$; if $r(t) \geq \bar{r}$ it is optimal to produce only $y$; and if $\underline{r} \leq r(t) \leq \bar{r}$ it is optimal to produce both goods.

Under the above assumptions the maximization utility problem is stated as follows:

$$
\begin{aligned}
& \text { Maximize } \int_{0}^{\infty} e^{-\rho t} V(Y(L, s(t) \mid p(t))) \mathrm{d} t, \\
& \text { subject to }\left\{\begin{array}{l}
L \leq \bar{L}, \\
\dot{R}(t)=-r(t), \\
R(t)>0, \quad \text { (predetermined), } \\
R(0)>0, \quad \text { (a given initial stock), } \\
r(t) \geq 0 .
\end{array}\right.
\end{aligned}
$$

The Hamiltonian, for an interior solution, is given by

$$
\dot{H}(\bar{L}, r(t), \lambda)=e^{-\rho t} V(Y(r(t), s(t) \mid p(t)))-\lambda r(t) .
$$

The first order condition is given by (see Appendix):

$$
\frac{\partial V}{\partial Y} \frac{\partial Y}{\partial r}=\lambda e^{\rho t}
$$


here $\lambda$ is a posive constant and this expression says that the present value of the marginal social product of the Hotelling factor is constant.

Lemma 5.1 Under the given assumptions, if $Y(t)$ has a Cobb-Douglas specification, say, $Y(t)=L^{1-\alpha} r(t)^{\alpha}$. Then the extraction declines at the rate

$$
-\frac{\rho}{1-\alpha(1+\eta)}
$$

where

$$
\eta=\frac{\partial^{2} V}{\partial Y^{2}} \frac{Y}{\frac{\partial V}{\partial Y}}
$$

is the elasticity of marginal utility of $\partial V / \partial Y$. That is,

$$
\frac{\dot{r}(t)}{r(t)}=\frac{\rho}{\eta}
$$

Consider the first order condition

$$
\frac{\partial V}{\partial Y} \frac{\partial Y}{\partial r}=\lambda e^{\rho t}
$$

and notice that

$$
\frac{\partial^{2} V}{\partial Y^{2}}\left(\frac{\partial Y}{\partial r} \dot{r}\right) \frac{\partial Y}{\partial r}+\frac{\partial V}{\partial Y} \frac{\partial^{2} Y}{\partial r^{2}} \dot{r}=\rho \lambda_{1} e^{-\rho t}=\rho \frac{\partial U}{\partial Y} \frac{\partial Y}{\partial r}
$$

therefore

$$
\frac{\partial^{2} V}{\partial Y^{2}} \frac{\dot{r}}{r} Y \alpha \frac{\partial Y}{\partial r}+\frac{\partial V}{\partial Y} \frac{\partial^{2} Y}{\partial r^{2}} \dot{r}=\frac{\partial Y}{\partial r} \rho \lambda_{1} e^{-\rho t}=\rho \frac{\partial V}{\partial Y} \frac{\partial Y}{\partial r}
$$

or

$$
\eta \alpha \frac{\dot{r}}{r}-(1-\alpha) \frac{\dot{r}}{r}=\rho
$$

equivalently

$$
\frac{\dot{r}}{r}=\frac{\rho}{\alpha(\eta+1)-1} .
$$

We also notice from the first order condition that:

Lemma 5.2 If $\eta$ is constant, then the rate of optimal path of rate of extraction is independent of the Ricardian factor endowment.

From the two previous lemmas it is easy to show:

Theorem 5.1 Ribzcynski Theorem for one Hotelling factor and one Ricardian factor. Let both goods be produced along an optimal trajectory, and let the same be true after an increase in $\bar{L}$. Suppose that $x$ is relatively intensive in $L$. Then, from the standard Rybczynski theorem, at each point in time where production takes place, there is an increase in the relative and absolute output of $x$. 
Other versions to the Stolper-Samuelson, Hecksher-Ohlin-Samuelson and Factor Price Equalization theorems remain valid.

\section{Renewable Factors}

For a small country that buys and sells at a given constant terms of trade, $p$, and able to borrow and lend at a given positive and constant interest rate $i$, then the problem to be solved, in compact form, may be stated as follows:

$$
\begin{gathered}
\text { Maximize } \int_{0}^{\infty} e^{-i t} Y(r(t)) \mathrm{d} t, \\
\text { subject to }\left\{\begin{array}{l}
\dot{R}(t)=-r(t)+G(R(t)), \\
R(0)>0, \\
r(t)>0,
\end{array}\right.
\end{gathered}
$$

where $R(t)>0, G(0)=0$ and $G^{\prime}(R)=d G(R) / d R>0$. Here, $R(t)$ is the state variable and $r(t)$ is the control variable. By using the Hamiltonian

$$
H(R(t), r(t))=Y(r(t))+\lambda(t) \dot{R}+\dot{\lambda}(t) R(t),
$$

where $\lambda(t)$ is the co-state variable, along with the first order conditions (see Appendix)

$$
\frac{\partial H}{\partial r}=0, \quad \text { and } \quad \frac{\partial H}{\partial R}=0,
$$

we get

$$
\left\{\begin{array}{l}
\frac{\partial Y}{\partial r} e^{-i t}-\lambda(t)=0, \\
G^{\prime}(R) \lambda(t)+\dot{\lambda}(t)=0 .
\end{array}\right.
$$

From the first condition, we obtain

$$
\dot{\lambda}(t)=\frac{\partial^{2} Y}{\partial r^{2}} \dot{r} e^{-i t}-i \frac{\partial Y}{\partial r} e^{-i t} .
$$

By using the second condition we find

$$
\dot{\lambda}(t)=-G^{\prime}(R) \frac{\partial Y}{\partial r} e^{-i t},
$$

so

$$
-G^{\prime}(R) \frac{\partial Y}{\partial r} e^{-i t}=\frac{\partial^{2} Y}{\partial r^{2}} \dot{r} e^{-i t}-i \frac{\partial Y}{\partial r} e^{-i t}
$$

hence

$$
-G^{\prime}(R)=\zeta \frac{\dot{r}}{r}-i
$$

where

$$
\zeta=\frac{\partial^{2} Y}{\partial r^{2}} \frac{r}{\frac{\partial Y}{\partial r}}
$$


is the elasticity of marginal product.

Theorem 6.1 The system of equations governing the dynamic in the $(R, r)$-phase space is given by

$$
\left\{\begin{array}{l}
\frac{\dot{r}}{r}=\frac{d}{\mathrm{~d} t} \log (r(t))=\frac{1}{\zeta}\left(i-G^{\prime}(R)\right), \\
\dot{R}(t)=-r+G(R) .
\end{array}\right.
$$

The steady state equilibrium for which $\dot{r}=0$ and $\dot{R}=0$ satisfies

$$
\left\{\begin{array}{l}
i=G^{\prime}(R), \\
r=G(R),
\end{array}\right.
$$

so the interest rate is matched by the rate of renewal and extraction matches the renewal.

We may also obtain some results in the $(R, \lambda)$-phase space. In such a case, the Hamiltonian is

$$
H(R, r)=Y(r)+\phi(-r+G(R)) .
$$

The first order conditions are $H_{r}=0$ and $H_{R}=i \phi-\phi$, or equivalently

$$
\left\{\begin{array}{l}
\frac{\partial Y}{\partial r}=\phi(t), \\
\dot{\phi}(t)=\left(i-G^{\prime}(R)\right) \phi(t) .
\end{array}\right.
$$

We have the following result:

Theorem 6.2 The system of equations governing the dynamic in the $(R, \phi)$-phase space is given by

$$
\left\{\begin{array}{l}
\dot{R}(t)=-r(t)+G(R), \\
\dot{\phi}(t)=\left(i-G^{\prime}(R)\right) \phi(t) .
\end{array}\right.
$$

The steady state equilibrium for which $\dot{R}=0$ and $\dot{\phi}=0$ satisfies:

$$
\left\{\begin{array}{l}
i=G^{\prime}(R), \\
r=G(R) .
\end{array}\right.
$$

Let us analyse now the case in which it is not possible to have access to perfectly competitive international capital markets, so the task is

$$
\begin{aligned}
& \text { Maximize } \int_{0}^{\infty} e^{-p t} V(Y(r(t))) \mathrm{d} t, \\
& \text { subject to }\left\{\begin{array}{l}
\dot{R}(t)=-r(t)+G(R(t)), \\
R(0)>0, \\
r(t)>0,
\end{array}\right.
\end{aligned}
$$


where $R(t)$ satisfies the same assumptions as before. By using the Hamiltonian

$$
H(R(t), r(t))=V(Y(r(t)))+\lambda(t) \dot{R}+\dot{\lambda}(t) R(t),
$$

the first order conditions are

$$
\left\{\begin{array}{l}
\frac{\partial V}{\partial Y} \frac{\partial Y}{\partial r} e^{-\rho t}-\lambda(t)=0 \\
G^{\prime}(R) \lambda(t)+\dot{\lambda}(t)=0
\end{array}\right.
$$

From the first condition we have

$$
\dot{\lambda}(t)=\frac{\partial^{2} V}{\partial Y^{2}}\left(\frac{\partial Y}{\partial r}\right)^{2} \dot{r} e^{-\rho t}+\frac{\partial V}{\partial Y} \frac{\partial^{2} Y}{\partial r^{2}} \dot{r} e^{-\rho t}-\rho \frac{\partial V}{\partial Y} \frac{\partial Y}{\partial r} e^{-\rho t} .
$$

By using the second condition we find

$$
\dot{\lambda}(t)=-G^{\prime}(R) \frac{\partial V}{\partial Y} \frac{\partial Y}{\partial r} e^{-i t}
$$

so

$$
-G^{\prime}(R) \frac{\partial V}{\partial Y} \frac{\partial Y}{\partial r} e^{-i t}=\frac{\partial^{2} V}{\partial Y^{2}}\left(\frac{\partial Y}{\partial r}\right)^{2} \dot{r} e^{-\rho t}+\frac{\partial V}{\partial Y} \frac{\partial^{2} Y}{\partial r^{2}} \dot{r} e^{-\rho t}-\rho \frac{\partial V}{\partial Y} \frac{\partial Y}{\partial r} e^{-\rho t}
$$

Therefore,

$$
-G^{\prime}(R)=(\epsilon+\zeta) \frac{\dot{r}}{r}-i
$$

where

$$
\epsilon=\frac{\partial Y}{\partial r} \frac{r}{Y}
$$

is the elasticity of output with respect to $r$.

Theorem 6.3 The system of equations governing the dynamic in the $(R, r)$-phase space is given by

$$
\left\{\begin{array}{l}
\frac{\dot{r}}{r}=\frac{1}{\epsilon+\zeta}\left(i-G^{\prime}(R)\right), \\
\dot{R}(t)=-r+G(R) .
\end{array}\right.
$$

The steady state equilibrium for which $\dot{r}=0$ and $\dot{R}=0$ can be obtained as before. We may also obtain some results in the $(R, \lambda)$-phase space. In such a case, the Hamiltonian is

$$
H(R, r)=V(Y(r))+\phi(-r+G(R)),
$$

the first order conditions are $H_{r}=0$ and $H_{R}=i \phi-\phi$, or equivalently

$$
\left\{\begin{array}{l}
\frac{\partial V}{\partial Y} \frac{\partial Y}{\partial r}=\phi(t) \\
\dot{\phi}(t)=\left(\rho-G^{\prime}(R)\right) \phi(t)
\end{array}\right.
$$


We also have the following result:

Theorem 6.4 The system of equations governing the dynamic in the $(R, \phi)$-phase space is given by

$$
\left\{\begin{array}{l}
\dot{R}(t)=-r(t)+G(R), \\
\dot{\phi}(t)=\left(\rho-G^{\prime}(R)\right) \phi(t),
\end{array}\right.
$$

The steady state equilibrium for which $\dot{R}=0$ and $\dot{\phi}=0$ satisfies

$$
\left\{\begin{array}{l}
\rho=G^{\prime}(R), \\
r=G(R),
\end{array}\right.
$$

that is the rate of time preference matches the rate of renewal. We may therefore conclude that:

Theorem 6.5 Rybczynski theorem for renewable factors. Let $R(0)$ be such that along an optimal path each commodity is produced during some interval. Then any increase in $R(0)$ raises the output of the $R$ intensive commodity.

\section{A Stochastic Model for Hotelling Factors}

We suppose again a small country able to buy and sell at a given constant terms of trade, $p$, and able to borrow and lend at a given positive and constant interest rate $i$, then the problem to be solved, in compact form, may be stated as follows:

$$
\begin{gathered}
\text { Maximize } E\left\{\int_{0}^{\infty} e^{-i t} Y(r(t)) \mathrm{d} t\right\} \\
\text { subject to }\left\{\begin{array}{l}
d R(t)=[-r(t)+G(R(t))] \mathrm{d} t+\sigma \mathrm{d} z(t), \\
R(0)>0, \\
r(t) \geq 0,
\end{array}\right.
\end{gathered}
$$

where $R(t)>0, G(0)=0$ and $\left.G^{\prime}(R)\right)=d G(R) / d R>0$. Here, $R(t)$ is the state variable, $r(t)$ is the control variable, $\mathrm{d} z(t)$ is a Brownian motion, and $\sigma^{2}$ is the instantaneous variance. That is, $\mathrm{d} z(t) \sim \mathcal{N}(0, \mathrm{~d} t)$. It is important to notice that when $\sigma=0$, we are in the case of exhaustible and renewable factors, and when $\sigma=0$ and $G=0$, we are in the case of exhaustible, but non-renewable factors.

In this stochastic extension, factors and extraction rates behave following a given pattern, namely, $\dot{R}(t)=-r(t)+G(t)$, but stochastic disturbances may occur through the term $\mathrm{d} z(t)$. These disturbances may be: market shocks, unexpected increases or decreases in oil reserves, or earthquakes or floods. Let us define

$$
\begin{gathered}
\mathcal{V}(R, t)=\max _{r} E\left\{\int_{t}^{\infty} e^{-i(s-t)} Y(r(s)) \mathrm{d} s \mid R, t\right\} \\
=E_{R, t}\left\{\int_{t}^{\infty} e^{-i(s-t)} Y(r(s)) \mathrm{d} s\right\} .
\end{gathered}
$$


Notice first that using Taylor's expansion we may write, $\mathrm{d} \mathcal{V}(R, t)=\frac{\partial \mathcal{V}}{\partial R} \mathrm{~d} R+\frac{\partial \mathcal{V}}{\partial t} \mathrm{~d} t+\frac{1}{2}\left[\frac{\partial^{2} \mathcal{V}}{\partial R^{2}}(\mathrm{~d} R)^{2}+2 \frac{\partial \mathcal{V}^{2}}{\partial R \partial t}(\mathrm{~d} R)(\mathrm{d} t)+\frac{\partial^{2} \mathcal{V}}{\partial t^{2}}(\mathrm{~d} t)^{2}\right]+o(\mathrm{~d} t)$

The operational rules of stochastic calculus are:

$$
\left\{\begin{array}{l}
(\mathrm{d} z)^{2}=\mathrm{d} t \\
(\mathrm{~d} t)^{2}=0 \\
(\mathrm{~d} t)(\mathrm{d} z)=0
\end{array}\right.
$$

Thus

$$
\mathrm{d} \mathcal{V}(R, t)=\left(\frac{\partial \mathcal{V}}{\partial R}[-r+G(R)]+\frac{\partial \mathcal{V}}{\partial t}+\frac{1}{2} \frac{\partial \mathcal{V}^{2}}{\partial R^{2}} \sigma^{2}\right)+\frac{\partial \mathcal{V}}{\partial R} \sigma \mathrm{d} z
$$

or

$\mathcal{V}(R, t)-\mathcal{V}(R, 0)=\int_{0}^{t}\left(\frac{\partial \mathcal{V}}{\partial R}[-r+G(R)]+\frac{\partial \mathcal{V}}{\partial s}+\frac{1}{2} \frac{\partial \mathcal{V}^{2}}{\partial R^{2}} \sigma^{2}\right) \mathrm{d} s+\int_{0}^{t} \frac{\partial \mathcal{V}}{\partial R} \sigma \mathrm{d} z \mathrm{~d} s$,

but $\mathrm{d} z \mathrm{~d} s=0$, so

$$
\frac{E_{R, 0}\{\mathcal{V}(R, t)\}-\mathcal{V}(R, 0)}{t}=E_{R, 0}\left\{\frac{1}{t} \int_{0}^{t}\left(\frac{\partial \mathcal{V}}{\partial R}[-r+G(R)]+\frac{\partial \mathcal{V}}{\partial s}+\frac{1}{2} \frac{\partial \mathcal{V}^{2}}{\partial R^{2}} \sigma^{2}\right) \mathrm{d} s\right\}
$$

Hence

$$
\lim _{t \rightarrow 0} \frac{E_{R, 0}\{\mathcal{V}(R, t)\}-\mathcal{V}(R, 0)}{t}=\frac{\partial \mathcal{V}}{\partial R}[-r+G(R)]+\frac{1}{2} \frac{\partial \mathcal{V}^{2}}{\partial R^{2}} \sigma^{2}
$$

since

$$
E_{R, 0}\left\{\frac{1}{t} \int_{0}^{t} \frac{\partial}{\partial s} \mathcal{V}(R, s) \mathrm{d} s\right\}=0
$$

The recursive Bellman equation is obtained as follows:

$$
\begin{aligned}
\mathcal{V}(R, 0) & =\max _{r} E_{R, 0}\left\{\int_{0}^{\infty} e^{-i s} Y(r(s)) \mathrm{d} s\right\} \\
& =\max _{r} E_{R, 0}\left\{\int_{0}^{t} e^{-i s} Y(r(s)) \mathrm{d} s+\int_{t}^{\infty} e^{-i s} Y(r(s)) \mathrm{d} s\right\} \\
& =\max _{r} E_{R, 0}\left\{\int_{0}^{t} e^{-i s} Y(r(s)) \mathrm{d} s+e^{i t} E_{R, t}\left\{\int_{t}^{\infty} e^{-i(s-t)} Y(r(s)) \mathrm{d} s\right\}\right\} \\
& =\max _{r} E_{R, 0}\left\{\int_{0}^{t} e^{-i s} Y(r(s)) \mathrm{d} s+e^{i t} \mathcal{V}(R, t)\right\} .
\end{aligned}
$$


Therefore,

$$
0=\max _{r}\left\{E_{R, 0}\left\{\int_{0}^{t} e^{-i s} Y(r(s)) \mathrm{d} s\right\}+e^{i t} E_{R, 0}\{\mathcal{V}(R, t)\}-\mathcal{V}(R, 0)\right\},
$$

equivalently

$$
\begin{aligned}
0 & =\max _{r}\left\{E_{R, 0}\left\{\frac{1}{t} \int_{0}^{t} e^{-i s} Y(r(s)) \mathrm{d} s\right\}+\frac{\left(e^{i t}-1\right)}{t} E_{R, 0}\{\mathcal{V}(R, t)\}\right. \\
& \left.+\frac{E_{R, 0}\{\mathcal{V}(R, t)\}-\mathcal{V}(R, 0)}{t}\right\},
\end{aligned}
$$

by letting $t \rightarrow 0$, we find

$$
0=\max _{r}\left\{Y(r)-i \mathcal{V}+\frac{\partial \mathcal{V}}{\partial R}[-r+G(R)]+\frac{1}{2} \frac{\partial^{2} \mathcal{V}}{\partial R^{2}} \sigma^{2}\right\}
$$

since by a simple application of L'hospital's rule

$$
\lim _{t \rightarrow 0} \frac{\left(e^{-i t}-1\right)}{t}=-i .
$$

Suppose $r$ is an optimal control then

$$
0=Y(r)-i \mathcal{V}+\frac{\partial \mathcal{V}}{\partial R}[-r+G(R)]+\frac{1}{2} \frac{\partial^{2} \mathcal{V}}{\partial R^{2}} \sigma^{2}
$$

taking the partial derivative with respect to $R$, we obtain

$$
0=-i \frac{\partial \mathcal{V}}{\partial R}+\frac{\partial^{2} \mathcal{V}}{\partial R^{2}}[r-G(R)]+\frac{1}{2} \frac{\partial^{3} \mathcal{V}}{\partial R^{3}} \sigma^{2}
$$

The first order condition is given by

$$
0=\frac{\partial Y}{\partial r}+\frac{\partial \mathcal{V}}{\partial R}
$$

if we suppose that $r=h(R)$, then

$$
\left\{\begin{array}{l}
\frac{\partial^{2} \mathcal{V}}{\partial R^{2}}=-\frac{\partial^{2} Y}{\partial r^{2}} \frac{\partial h}{\partial r} \\
\frac{\partial^{3} \mathcal{V}}{\partial R^{3}}=-\frac{\partial^{3} Y}{\partial r^{3}}\left(\frac{\partial h}{\partial r}\right)^{2}-\frac{\partial^{2} Y}{\partial r^{2}} \frac{\partial^{2} h}{\partial R^{2}}
\end{array}\right.
$$

Therefore,

$$
0=\frac{\partial^{2} Y}{\partial r^{2}} h^{\prime}[-r+G(r)]+\frac{\partial Y}{\partial r}\left[G^{\prime}(R)-i\right]+\frac{1}{2} \sigma^{2} \frac{\partial^{3} Y}{\partial r^{3}}\left(h^{\prime}\right)^{2}+\frac{\partial^{2} Y}{\partial r^{2}} h^{\prime \prime} .
$$


As a conclusion we have the following:

Theorem 7.1 Under de assumption of a small country able to buy and sell at a given constant terms of trade, $p$, and able to borrow and lend at a given positive and constant interest rate $i$, whose task is to to solve:

$$
\begin{aligned}
& \text { Maximize } E\left\{\int_{0}^{\infty} e^{-i t} Y(r(t)) \mathrm{d} t\right\}, \\
& \text { subject to }\left\{\begin{array}{l}
d R(t)=[-r(t)+G(R(t))] \mathrm{d} t+\sigma \mathrm{d} z(t), \\
R(0)>0, \\
r(t) \geq 0,
\end{array}\right.
\end{aligned}
$$

where $R(t)>0, G(0)=0$ and $\left.G^{\prime}(R)\right)=d G(R) / d R>0$. Then,

(i) As long as $r=h(R)=$ constant, then $i=G^{\prime}(R)$, that is, the interest rate is matched with the rate of renewal.

(ii) As long as $r=h(R)$ is linear, that is $r=a R+b$, then

$$
0=\frac{\partial^{2} Y}{\partial r^{2}} a[-r+G(r)]+\frac{\partial Y}{\partial r}\left[G^{\prime}(R)-i\right]-\frac{1}{2} \sigma^{2} \frac{\partial^{3} Y}{\partial r^{3}} a^{2},
$$

which is a third order partial differential equation. If $Y$ is Cobb-Douglas with one Ricardian factor $\bar{L}$, say, $Y=\bar{L}^{\alpha} r^{1-\alpha}$. Then

$$
0=r^{2}\left(G^{\prime}(R)-i+a \alpha\right)-\alpha a G(R)+\frac{1}{2}(1+\alpha) \alpha a^{2} \sigma^{2},
$$

and the condition for real solutions in the above quadratic equation is

$$
G^{\prime}(R)-i \leq \frac{1}{2}\left(\frac{G(R)}{\sigma}\right)^{2} \frac{\alpha}{1+\alpha}-a \alpha=M(R \mid \alpha, a, \sigma) .
$$

That is, the difference between the rates of renewal and interest remain below $M$.

For case (i) Ribczynski theorem survives, however since output can be specialized at all $t$, there is not a close analogue of the Stolper-Samuelson theorem. Case (ii) deserves more attention and additional assumptions have to be made to get close analogues to Rybczynski and Hecksher-Ollin-Samuelson theorems.

\section{Final Comments}

We have presented in this paper a number of dynamic and stochastic extensions to the main theorems in international trade (Ribczynski, Stolper-Samuelson, Hecksher-Ohlin-Samuelson and Factor Price Equalization). We consider exhaustible and non-renewable or renewable resources. In our extensions, stochastic differential equations and Brownian motion have played an important role. The well-known deterministic cases become particular cases of our approach. 
Of course, more work has to be done for the stochastic case, so more and much better results can be obtained.

We mention as a delimitation of our work that the case of resource-replacement is missing, for instance, when factors can be oil and solar energy, however, this is already included in our future tasks.

A more interesting problem to work with, in the future, is:

$$
\begin{gathered}
\text { Maximize } \int_{0}^{\infty} e^{-\rho t} V(Y(K, L, s(t) \mid p(t))) \mathrm{d} t, \\
\text { subject to }\left\{\begin{array}{l}
d L=n L \mathrm{~d} t+\sigma_{1} \mathrm{~d} z_{1}, \\
d R(t)=[-r(t)+G(R)] \mathrm{d} t+\sigma_{2} \mathrm{~d} z_{2}, \\
d K(t)=Y(K, L, s(t)) \mathrm{d} t+\sigma_{3} \mathrm{~d} z_{3}, \\
R(t) \geq 0, K(t) \geq 0 \text { (predetermined), } \\
R(0)>0, K(0)>0, L(0)>0, \\
r(t) \geq 0,
\end{array}\right.
\end{gathered}
$$

where population follows the differential stochastic equation of exponential growth.

A very interesting point in the above model is the connection between economic growth and international trade. This dynamic and stochastic approach will provide a formal and systematic analysis of capital acumulation in the context of a Ramsey problem in open economies. The dynamics of the capitalto-labor ratio are described by a diffusion type stochastic process, there are several sources of uncertainty: in the population size; in the availability of a renewable resource; and in investment. We want to find the asymptotic distributions for most of the economic variables involved. We also want to show that in the special case of a Cobb-Douglas production function with a constant savings function, a version of Ribczinski theorem will survive.

\section{Appendix}

The maximization utility problems stated through sections 4 to 7 in this work may be solved by using: (I) Calculus of variations (Euler-Lagrange equations) when a constraint in the form of of a differential equation is transformed into an integral form; or (II) Optimal control (Pontryagin maximum principle).

The following problems are equivalent, some of them take into account Lagrangians $(L)$ and discounted Hamiltonians $(H)$ :

$$
\left\{\begin{array}{l}
\text { Maximize } \int_{0}^{\infty} V(Y(r(t))) e^{-\rho t} \mathrm{~d} t, \\
\text { subject to } \int_{0}^{\infty} B(Y(t), R(t), r(t)) e^{-i t} \mathrm{~d} t=\text { constant, } \\
L(Y(r(t)), R(t), r(t), \lambda)=\{V(Y(r(t)))+\lambda B(Y(t), R(t), r(t))\} e^{-i t}, \\
\text { F.O.C. } L_{r}=0, \quad \rho=i .
\end{array}\right.
$$



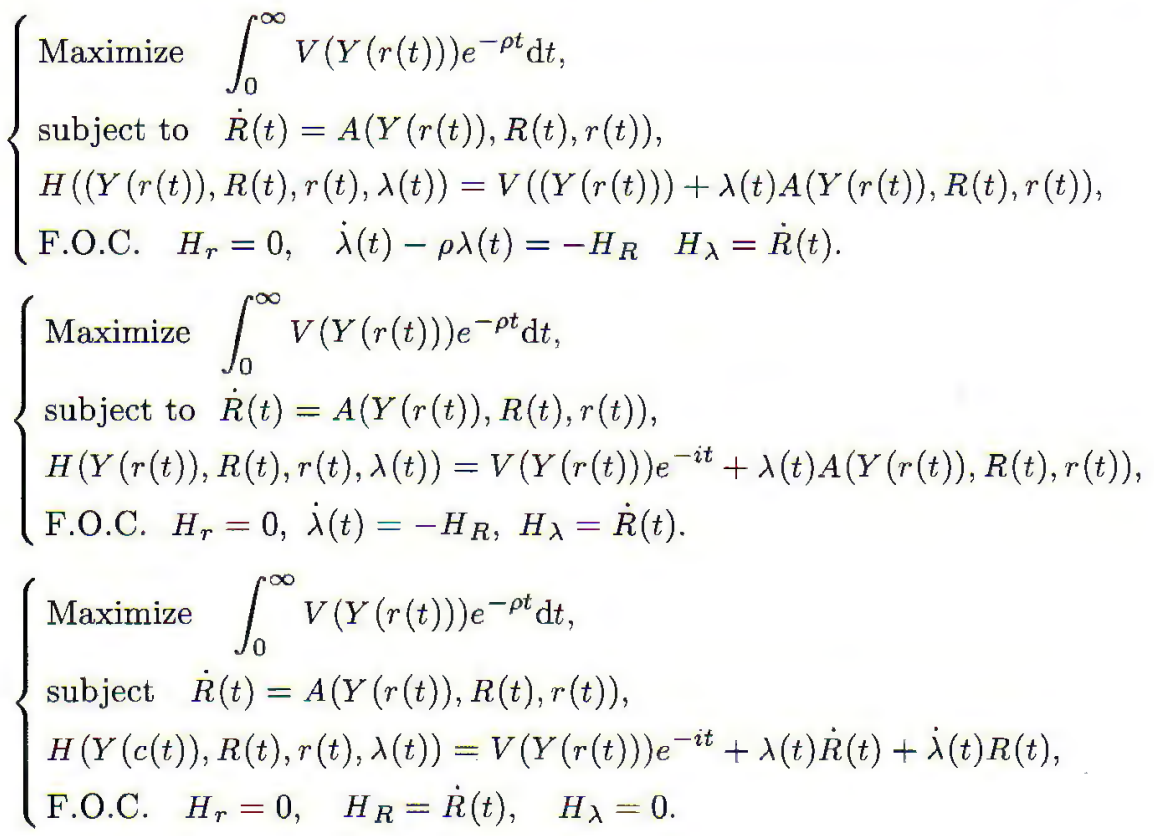

\section{References}

Kemp, M. C. and N. V. Long. (1982), Rybczynski's theorem in a context of exhaustible resources: The case of time-contingent prices, International Economic Review, Vol. 23, No. 3, pp. 699-710.

Kemp, M. C. and N. V. Long. (1979), International trade with an exhaustible resource: $A$ theorem of Rybcynski type, International Economic Review, Vol. 20, No. 3, pp. 671-677.

Tawada, M. (1982), A note on international trade with a renewable resource, International Economic Review, Vol. 23, No. 1, pp. 157-163. 